\title{
Socioeconomic background modulates cognition-achievement relationships in reading
}

\author{
Kimberly G. Noble ${ }^{\mathrm{a}, \mathrm{b}, *}$, Martha J. Farah ${ }^{\mathrm{a}}$, Bruce D. McCandliss ${ }^{\mathrm{b}}$ \\ ${ }^{\text {a } C e n t e r ~ f o r ~ C o g n i t i v e ~ N e u r o s c i e n c e, ~ U n i v e r s i t y ~ o f ~ P e n n s y l v a n i a, ~} 3720$ Walnut Street, Philadelphia, PA 19104 USA \\ b Sackler Institute for Developmental Psychobiology, Weill Medical College of Cornell University, \\ 1300 York Avenue, Box 140, New York, NY 10021, USA
}

\begin{abstract}
Multiple factors contribute to individual differences in reading ability. The two most thoroughly examined are socioeconomic status (SES) and phonological awareness (PA). Although these factors are often investigated individually, they are rarely considered together. Here we propose that SES systematically influences the relationship between PA and reading ability, and test this prediction in 150 first-graders of varying SES and PA. Results confirm a multiplicative relationship between SES and PA in decoding skills, such that decreased access to resources may amplify cognitive risk factors for poor decoding, whereas greater access to resources may buffer reading skills among children with weaker PA. Attempts to identify the cognitive and experiential factors driving development must acknowledge the complex, synergizing relations between these factors.
\end{abstract}

(C) 2006 Elsevier Inc. All rights reserved.

Keywords: Cognition; Socioeconomic status; Phonological awareness; Reading; Language

Many factors contribute to individual differences in early childhood reading ability. To optimize reading education strategies, we must understand how these factors relate to and interact with each other in the development of reading skills. Extensive literatures have addressed both the cognitive and social correlates of reading development. Sociological research has provided insights into the relationship between reading achievement and a child's socioeconomic background, whereas the traditions of cognitive and educational psychology have largely focused on the cognitive precursors to reading ability. However, although a number of studies have examined the distinct associations of either socioeconomic status (SES, e.g. R.H. Bradley, Corwyn, Burchinal, Pipes McAdoo, \& Garcia Coll, 2001; White, 1982; Whitehurst, 1997) or phonological awareness (PA,

\footnotetext{
* Corresponding author. Tel.: +1 212746 5837; fax: +1 2127465755.

E-mail address: kimnoble@med.upenn.edu (K.G. Noble).
} 
e.g. Adams, 1990; L. Bradley \& Bryant, 1983; Wagner \& Torgesen, 1987) with reading development, surprisingly few have considered these two factors together (Bowey, 1995; Hecht, Burgess, Torgesen, Wagner, \& Rashotte, 2000; Raz \& Bryant, 1990), and none has examined how a child's social background may interact with PA in predicting reading ability. Such approaches implicitly make strong assumptions about the independence of these two factors. Here we hypothesize that these two factors are not independent, but rather, that a child's background and experiences are likely to directly interact with the cognitive precursors to reading. This "Multiplicative Factors Hypothesis," outlined below, predicts that the relationship between PA and reading achievement will be systematically modulated by SES.

SES is strongly associated with many measures of childhood cognitive and academic achievement, including IQ (Liaw \& Brooks-Gunn, 1994; Smith, Brooks-Gunn, \& Klebanov, 1997), achievement test scores (Brooks-Gunn, Guo, \& Furstenberg, 1993), grade retentions and functional literacy (Baydar, Brooks-Gunn, \& Furstenberg, 1993). These effects are typically quite substantial: in one study, SES was found to account for approximately $20 \%$ of the variance in childhood IQ (Gottfried, Gottfried, Bathurst, Guerin, \& Parramore, 2003). Among such broad barometers of achievement, SES has been found to have particularly large effects within the relatively specific domain of language development (Noble, Norman, \& Farah, 2005; Whitehurst, 1997; Hoff, 2003). Reading development is an aspect of language skill that bears a strong influence on academic and life achievement; correlations between SES and word reading ability typically fall within the range of 0.3-0.7 (White, 1982), and evidence has suggested that SES is predictive both of decoding single words and non-words, as well as reading comprehension (Bowey, 1995; Hecht et al., 2000; Raz \& Bryant, 1990).

Although SES is most commonly indexed using education, occupation, and income (Ensminger \& Fothergill, 2003), many other factors vary systematically with SES (Barnett, 1998; BrooksGunn, Klebanov, \& Duncan, 1996; Hawley \& Disney, 1992; Jackson, Brooks-Gunn, Huang, \& Glassman, 2000; Klein, Hack, \& Breslau, 1989; Korenman, Miller, \& Sjaastad, 1995; Leventhal \& Brooks-Gunn, 2003b; McCormick, 1989; Needleman, Schell, Bellinger, Leviton, \& Allred, 1990; Ramey \& Ramey, 1998) and are likely to play a role in creating and sustaining the SES gap in cognitive performance and achievement (Bornstein \& Bradley, 2003). For this reason the association between SES and reading ability is likely to be multifactorial. Nevertheless, it is often suggested that a major determinant of the relationship between SES and reading ability is the SES gradient in reading-related experiences, such as the home literacy environment, degree of early print exposure, and quality of early schooling (Hecht et al., 2000). Two types of findings support this view.

First, there is a substantial and robust SES gradient in children's reading-related experience. Both the number of hours parents spend reading with their children and the number of books in the home are positively associated with SES, especially prior to school age (Raz \& Bryant, 1990; Whitehurst, 1997). Across ethnic groups, children from higher SES backgrounds are far more likely to own books and to have greater access to resources such as museums or libraries (R.H. Bradley, Corwyn, Pipes McAdoo, \& Garcia Coll, 2001). Second, the amount of reading-related experience is predictive of reading ability. Significant correlations have been noted between the frequency of shared book reading in the home and preschoolers' language abilities (Whitehurst, 1997), and a strong relation between home learning environment and reading skill has been demonstrated (R.H. Bradley, Corwyn, Burchinal et al.). SES has thus been repeatedly shown to be associated with home literacy environment, which in turn is associated with reading achievement.

In a largely independent line of research, ample evidence has shown that PA, or an understanding of the sound structure of language, is fundamentally necessary for the successful acquisition of 
reading skill (e.g. Adams, 1990; L. Bradley \& Bryant, 1983; Wagner \& Torgesen, 1987). That is, the ability to analyze, synthesize and manipulate phonemes and syllables must be mastered in order for a child to adequately learn precisely how these sounds correspond to the arbitrarily defined shapes known as letters. Empirical evidence for this has come from the fact that preliterate children who have better PA skills are in turn quicker to learn to read (Wagner \& Torgesen, 1987), and that both the absolute level (Share, Jorm, MacLean, \& Mathews, 1984) and rate of acquisition (Byrne, Fielding-Barnesley, \& Ashley, 2000) of early PA skills are excellent predictors of elementary reading skills. Wagner et al. (1997) found that individual differences in PA persisted in explaining reading abilities each year from kindergarten through fourth grade, whereas the relationships between reading and other skills faded with development. In fact, kindergarten PA is a better predictor of teenage reading ability than is kindergarten reading skill (MacDonald \& Cornwall, 1995). Studies of twins have suggested that phonological skills have both genetically heritable and environmentally influenced components (Fisher \& DeFries, 2002; Knopnik et al., 2002), and a deficit in phonological processing is now believed to be the primary core deficit in developmental dyslexia, a reading disability in which children exhibit a difficulty in accurately or fluently reading at age-appropriate levels (L. Bradley \& Bryant, 1983). This phonological impairment is reflected in dysfunction of left perisylvian cortical regions during reading tasks (McCandliss \& Noble, 2003). Importantly, experimental manipulations in which reading-disabled children were exposed to both phonological skill training and explicit instruction in letter-sound correspondence rules have resulted in improved reading ability, relative to various reading-disabled control groups (L. Bradley, \& Bryant, 1983; Foorman, Francis, Novy, \& Liberman, 1991; Wagner \& Torgesen, 1987), suggesting that phonological skill is not merely associated with reading, but actually plays a causal role in its development. PA has been repeatedly associated with the ability to decode both words and non-words, and to a lesser extent, with reading comprehension ability (see Share, 1995 for a review). This well-established link between PA and reading skills has greatly influenced our understanding of both mainstream and remedial education practices, and has had a large impact on education policy (Report of the National Reading PanelReport of the National Reading Panel, 2000). It is imperative, however, that researchers and educators understand how a cognitive factor such as PA interacts with the background and experiences that a child brings to the classroom.

Despite abundant independent work showing that socioeconomic background and PA are each associated with reading achievement, surprisingly few studies have examined how SES relates to PA in predicting individual differences in reading ability. In studies of children's language or reading development, SES is often controlled for, in an implicit (or explicit) recognition of its association with the outcomes of interest (e.g. Wagner \& Torgesen, 1987). However, by matching groups or covarying out the effect of SES, one does not examine the unique variance it accounts for, thereby discounting its role and the role of variables for which it serves as a proxy as variables of interest. Although many studies have shown that SES and PA are associated (e.g. Dickinson \& Snow, 1987; Noble et al., 2005; Wallach, Wallach, Dozier, \& Kaplan, 1977; Whitehurst, 1997), the nature of this relationship is less clear.

We propose that a child's background and experiences are likely to directly influence the association between a child's understanding of language sounds and his or her ability to map these sounds onto letters. PA is a skill that varies from child to child; as detailed above, some individuals show difficulties with this skill, which in turn predict difficulties with reading. We hypothesize, however, that this predictive relationship varies as a function of access to resources. A child with higher PA skills is likely to succeed at reading, regardless of background. When a child has lower phonological skill, however, differences in socioeconomic background may come into play. Advantaged parents might have the resources to increase environmental exposures or seek out 
alternate educational strategies. In a highly supportive environment, such a child may thus be more likely to overcome phonological difficulty to develop adequate reading achievement. In contrast, less advantaged parents may be less likely to recognize low phonological skill or be able to provide the resources necessary to overcome such a difficulty. We therefore predict that the relationship between PA and reading ability will be attenuated when resources are plentiful, but amplified when resources are scarce. This possibility, termed the "Multiplicative Factors Hypothesis," states that SES modulates the association between PA and reading ability. Mechanistically, this model implies that, although PA skills are a cognitive precursor to reading ability, the strength of this relationship varies across the SES spectrum. Statistical evidence for this model would come in the form of an SES $\times$ PA interaction, such that the effect of PA on reading ability differs across the SES spectrum.

Of course, other relationships between PA, SES, and reading are possible as well. One alternative, the "Mediating Factor Model," suggests that PA directly influences reading ability, but that SES affects reading only indirectly through its impact on this cognitive precursor to reading. Mechanistically, this model states that differences in experiences associated with SES result in differences in the development of PA, but that it is these differences in PA that directly impact reading. If it is true that PA mediates the effect of SES on reading, then any variance in reading ability that is explained by SES could be entirely accounted for by PA. That is, when statistically (or experimentally) controlling for PA, no additional variance in reading ability would be attributable to SES. A second alternative, the "Independent Factors Model," holds that SES and PA each exert independent effects on reading ability. Mechanistically, this model proposes that reading is determined by a combination of PA and some other skills for which SES serves as a proxy, such as, for example, letter knowledge or print familiarity. In this case, some unique, non-overlapping variance in reading ability could be statistically accounted for by each factor.

Different reading tasks tap into different types of skills. Basic decoding of non-words is highly dependent on phonological skill. It is an excellent predictor of later reading ability, and is relatively unlikely to be confounded by vocabulary or word-frequency effects (Share, 1995). While other tasks involving single word reading or reading comprehension may be closer to real-life reading exercises, they are also more likely to be influenced by multiple, non-phonological factors. The predictions of the Multiplicative Factors Hypothesis, as well as those of the two alternate models described above, vary according to the reading test administered. Reading skills such as nonword reading that rely most heavily on phonological factors would provide the greatest power for revealing an SES $\times$ PA interaction under the Multiplicative Factors Hypothesis. In contrast, the Mediating Factors Model would predict that, regardless of the type of reading test administered, any effect of SES is always a result of differences in PA. Under the Independent Factors Model, the less a particular reading skill relies solely on phonological skills, the more room there might be for non-phonological factors to play a role. That is, under this model, SES might account for a small portion of the variance in a more phonologically "pure" skill such as non-word reading, while a greater portion of the variance might be accounted for by SES in a complex skill such as reading comprehension.

The existence of the predicted SES $\times$ PA interaction is potentially quite relevant both to the academic study of reading and to the design of educational intervention programs for reading disabled children. If socioeconomic background systematically impacts the relationship between PA and reading abilities, then a non-constant relation between PA and reading would be expected across the SES spectrum. In a given investigation, the population studied may directly influence the relative magnitude of the association between PA and reading. The presence of an interaction may therefore be responsible for conflicting results across studies; by neglecting to investigate 
the presence of an interaction, one could falsely misinterpret the lack of a main effect of SES. Furthermore, if an interaction exists, this information could potentially be used to predict how the efficacy of phonologically based intervention techniques might differ across children of different backgrounds. Examining the data for such an interaction effect is therefore imperative, both from a methodological and educational standpoint.

Certainly, it is possible that some combination of these three possibilities captures the nature of the actual relationship between SES, PA, and reading. Several studies have shed light on the possible means by which SES and PA together influence reading skills. The studies that support the various models are described below.

The Mediating Factors Model, suggesting that any association between SES and reading is entirely mediated by PA, has received some support. For instance, Raz and Bryant (1990) reported SES differences in word reading, but found that controlling for PA eliminated such differences. Similarly, Bowey (1995) found that SES differences on both word and non-word reading were eliminated after covarying PA, suggesting that SES may impact decoding via its effect on phonological skill.

The Independent Factors Model, suggesting that PA and SES each explain a unique portion of the variance in reading ability, was also supported in part by Raz and Bryant (1990). Although these authors found that PA mediated the association between SES and word reading, they reported that, after controlling for PA, a significant difference remained across SES in reading comprehension, implying that both PA and SES exert unique effects on this skill. Interestingly, Bowey (1995) found that controlling for verbal ability eliminated SES differences on reading comprehension, although SES associations with PA remained. Together, these results imply that verbal skills mediate the link between SES and reading comprehension, and are distinct from the association between SES and PA. Further support comes from Hecht et al. (2000), who found that SES continued to account for unique variance in both decoding and comprehension skills, even when PA, verbal abilities, prior decoding, rapid naming and print knowledge were taken into account. Together, these studies support the idea that PA and SES underscore unique aspects of reading.

Importantly, none of the aforementioned studies were designed to test the Multiplicative Factors Hypothesis. Although the studies examined whether the variance in reading ability accounted for by PA and SES is unique, none has examined whether these two factors interact. Does the effect of PA on reading ability vary, as we suggest, as a function of SES? A hint at such an interaction has been suggested by the work of Lonigan, Burgess, Anthony, and Barker (1998), who found that letter knowledge - which itself is associated with SES (Adams, 1990; Raz \& Bryant, 1990) - is more strongly related to PA in low SES preschoolers relative to their middle SES peers. However, it is not clear whether a similar interaction is seen when later-developing reading abilities are the dependent variables of interest. By investigating this question in young readers, we can more directly examine how PA influences reading skill across the SES spectrum, and can test for the possibility of a multiplicative relationship among risk factors for poor reading achievement.

Here, we investigate whether the relationship between PA and reading is consistent across the SES spectrum, or conversely, the extent to which an SES $\times$ PA interaction exists. We examine this question across three distinct reading measures: non-word reading, single word reading, and reading comprehension. Whereas non-word reading ability is the purest measure of phonological skill, the other reading measures are increasingly sensitive to non-phonological aspects of reading ability such as print familiarity and vocabulary, and thus may involve the use of other skills. As in previous studies (e.g. Raz \& Bryant, 1990), we explore the variance in reading accounted for by verbal ability. 


\section{Method}

\subsection{Participants}

One hundred sixty-eight 1st-graders were recruited from 9 New York public schools that serve families from a wide range of socioeconomic backgrounds, to participate in a larger study on neurocognitive performance. Parents signed IRB-approved permission slips for their children to engage in a short in-school battery of cognitive tests, for which their children would receive a free book. We were able to reach the parents of 150 of these children by telephone ( 80 boys, 70 girls) to answer a 5-min questionnaire. These 150 children represent the participants in the majority of analyses presented below. Thirty-four percent of children were identified by their parents as African-American; $7 \%$ were Asian; $23 \%$ were of Latin American descent; $23 \%$ were white; and $14 \%$ of children were identified as mixed or other. Twenty-seven percent of children came from single-parent homes. All children were native English speakers. Although in all cases English was the primary language spoken at home, 68 children grew up in a family in which another language was also spoken by at least one family member part of the time; results of relevant analyses accounting for second language exposure are presented below.

Five children weighed less than $1500 \mathrm{~g}$ at birth, and 18 were reported as having spent some time in the Neonatal Intensive Care Unit (NICU). Three children were reported to have ADHD, one of whom was taking Ritalin. No other child was taking psychotropic medications of any kind. No child had been diagnosed with a learning disability (although it should be noted that first grade is a bit early for a confident diagnosis). Fourteen children were reported to have a history of some other type of psychiatric or developmental problem. One child suffered a head injury involving loss of consciousness for several hours. Because physical and mental health factors could account for SES differences in cognitive performance (Hawley \& Disney, 1992; Klein et al., 1989; McCormick, 1989; Needleman et al., 1990), analyses will be presented below both including and excluding children with medical or psychiatric histories.

\subsection{Participants within the average PA range}

To properly test for the presence of an SES $\times$ PA interaction, it is necessary to ensure that a range of SES is represented across all levels of PA. However, in a non-referred community sample, SES and PA are themselves correlated to such an extent that the range of SES is significantly limited for those scoring in the lowest range of the PA measures (see results below). It was therefore necessary to restrict certain analyses to those children who scored within a range of phonological skill for which an adequate range of SES scores were collected. Children from a broad range of socioeconomic backgrounds scored within one standard deviation above and below the national mean on a composite of PA measures. These 116 children (61 girls, 55 boys) were demographically quite similar to the full sample: $28 \%$ were identified by their parents as African-American; $8 \%$ were Asian; 23\% were of Latin American descent; $27 \%$ were white; and 15\% of children were identified as mixed or other. Twenty-four percent of children came from single-parent homes. Fifty-six children from the subset grew up in a family in which another language was also spoken by at least one family member part of the time. Two children weighed less than $1500 \mathrm{~g}$ at birth, and 12 were reported as having spent some time in the NICU after birth. One child was reported to have a diagnosis of ADHD, and was taking Ritalin. No other child was taking psychotropic medications. Seven of these children's parents reported a history of some type of psychiatric or developmental problem. No children were reported to have suffered a head injury involving loss of 
consciousness. The subset of children who scored within the average PA range is used in analyses only where explicitly indicated.

\subsection{Procedures}

Participants were tested in a quiet location in school, as part of a larger testing battery. Testing occurred during the first grade, between March and June.

\subsubsection{Reading measures}

Tests came from WJIII Tests of Achievement (Woodcock, McGrew, \& Mather, 2001).

1.3.1.1. Word attack (non-word reading). Unfamiliar non-words, which must be decoded by letter-sound correspondence rules, are read. Items progress in difficulty and the test is terminated when ceiling is reached. Split-half reliability at the age level tested here ranges from 0.92 to 0.94 ; test-retest reliability is 0.79 .

1.3.1.2. Word ID (single word reading). High-frequency real words, many of which are not decodable by letter-sound correspondence rules, are read. Items progress in difficulty and the test is terminated when ceiling is reached. Split-half reliability at the age level tested here ranges from 0.97 to 0.98 ; test-retest reliability is 0.92 .

1.3.1.3. Passage comprehension. Children read short texts ranging from simple sentences to complex paragraphs, and respond to each by filling in a blank embedded in the text. Items progress in difficulty and the test is terminated when ceiling performance is reached. Split-half reliability at the age level tested here is 0.96 ; test-retest reliability is 0.89 .

\subsubsection{Phonological awareness measures}

Tests came from the comprehensive test of phonological processing (CTOPP, Wagner, Torgesen, \& Rashotte, 1999). Standard scores were averaged to formulate a PA composite. (Note that these two tests can be combined into a standardized phonological composite for ages 7 and up; this was not possible in our sample as not all children were yet 7 years old.)

1.3.2.1. Blending words. This 20 -item subtest measures the child's ability to combine sounds to form words. The child listens to a series of tape-recorded sounds and is asked to put the sounds together to make a whole word. Items progress in difficulty and the test is stopped when the child answers three items in a row incorrectly. The internal consistency at the age level tested here ranges from 0.86 to 0.89 , and the test-retest reliability of this subtest is 0.88 .

1.3.2.2. Elision. This 20 -item subtest measures the child's ability to say a word without saying a specific sound. For example, the child is told to say bold, and to then say bold without saying 'b'. Items progress in difficulty and the test is stopped upon three incorrect items in a row. The internal consistency at the age level tested here ranges from 0.90 to 0.92 , and the test-retest reliability of this subtest is 0.88 .

\subsubsection{Receptive vocabulary}

In The peabody picture vocabulary test, 3rd edition (PPVT-III), the child hears a word on each trial, and must select the corresponding picture from among four choices. The internal consistency at the age level tested here ranges from 0.93 to 0.95 , and the test-retest reliability is 0.93 . 


\subsubsection{Parent questionnaire}

Parents were administered a 5-min questionnaire involving questions pertaining to parental education, occupation, and income levels, as well as to the child's medical and psychiatric history. Parental education was defined as the average years of education of any parents, step-parents, or guardians in the home. Parental occupation was defined as the highest occupational score of any parent, step-parent, or guardian in the home, according to the 9-point Hollingshead Index Occupational Status Scale (Hollingshead, 1975, as cited in Bornstein \& Bradley, 2003). The income-to-needs ratio, defined as the total family income divided by the official poverty threshold for a family of that size, was calculated for each family, such that a family with an income-toneeds ratio of 1 is living at the poverty line (McLoyd, 1998). A composite SES score was then calculated for each child by entering parental education, occupation, and income-to-needs into a factor analysis, through which a single factor was extracted. This factor loading was used as the SES composite score for each child.

\section{Results}

\subsection{Calculation of SES composite}

A stable measure of SES incorporates education, occupation, and income (McLoyd, 1998). Although ideally all parents would provide data pertaining to all three components of SES, in reality, parents are often more willing to provide education and occupation than income data (Bornstein \& Bradley, 2003). Although 150 parents provided education and occupation information, only 130 of these were willing to disclose income. To avoid discarding the data from the remaining 20 children (and therefore potentially biasing results towards those families who, for whatever reason, were willing to provide income information), a regression equation was constructed to predict the income-to-needs ratio from the other two variables in the participants for whom we had all three variables. The nature of the income-to-needs ratio, however, affords the possibility of positive, but not negative, outliers: that is, all families living below the poverty line are distributed between values of 0 and 1, whereas very wealthy families may, in theory, be extreme outliers with very high income-to-needs ratios. To illustrate this point, the mean incometo-needs ratio in our sample of 130 parents who provided this information was 3.36 (S.D. 3.78); however, whereas the minimum ratio was only 0.23 (less than one standard deviation from the mean), the maximum was 19.5 (over 4 standard deviations from the mean). To eliminate the skewing effect that these positive outliers would have on predicting the missing data, we eliminated the 9 families who had income-to-needs ratios greater than 10, at which point the standardized residuals displayed a normal distribution. A regression equation was then calculated from the remaining 121 families (income-to-needs $=0.358$ (parental education) +0.344 (parental occupation) $-4.097 ; R^{2}=0.545 ; p<0.0001$ ); this equation was used to impute the income-to-needs scores for the 20 children whose parents did not provide income information, using the education and occupation information that those children's parents provided. An SES composite score was then determined for each child by entering the three variables (parental education, occupation, and income-to-needs or imputed income-to-needs) into a factor analysis, using the maximum likelihood method of extraction. A single factor was extracted, explaining $73.5 \%$ of the variance across the three variables. This factor loading was used as the SES composite score for each child. As this composite was based on a factor loading score, scores had a mean of 0 (range -1.7 to +2.7 ; S.D. 0.91 ). 
Table 1

Means and standard deviations of phonological awareness, vocabulary, and reading measures

\begin{tabular}{|c|c|c|c|c|}
\hline \multirow[t]{2}{*}{ Task } & \multicolumn{2}{|l|}{ Mean } & \multicolumn{2}{|c|}{ Standard deviation } \\
\hline & Whole sample & Average PA & Whole sample & Average PA \\
\hline CTOPP-blends ${ }^{\mathrm{a}}$ & 8.9 & 9.4 & 2.3 & 1.6 \\
\hline CTOPP-elision ${ }^{\mathrm{a}}$ & 9.2 & 9.8 & 3.2 & 2.3 \\
\hline PPVT $^{b}$ & 93.7 & 96 & 15 & 14 \\
\hline Non-word reading ${ }^{\mathrm{c}}$ & 101.2 & 105 & 15 & 12 \\
\hline Single word reading ${ }^{\mathrm{c}}$ & 105.4 & 109 & 15 & 12 \\
\hline Passage comprehension ${ }^{\mathrm{c}}$ & 98.8 & 100 & 11 & 9 \\
\hline
\end{tabular}

Note: All means and standard deviations represent standard scores. CTOPP: comprehensive test of phonological processing; PPVT: peabody picture vocabulary test, 3rd edition.

a Nationally normed mean age-standard score of 10, with a standard deviation of 3 .

b Nationally normed standard score of 100 , with a standard deviation of 15 , normed to age.

c Nationally normed standard score of 100 , with a standard deviation of 15 , normed to grade.

\subsection{Outliers and bivariate correlations}

A total of three individual task scores fell more than three standard deviations on either side of the mean of the sample and were eliminated from the data set (one high performance each on passage comprehension and PPVT, and one low performance on single word reading.) One additional child refused to complete the passage comprehension task. Table 1 shows the means and standard deviations of the remaining scores for each task for the whole group and the subset.

Before examining the additive or interactive nature of SES and PA in predicting reading skill, the extent to which these variables were related was first determined by examining the bivariate correlations. As expected, all were highly correlated. Table 2 demonstrates the correlations between SES, PA, non-word reading, single word reading, passage comprehension, and the PPVT.

\subsection{Regressions using SES composite scores}

\subsubsection{SES composite and PA}

Hierarchical regressions were conducted, entering PA, followed by SES. Table 3 shows that PA contributed unique variance in all three reading tasks, whereas SES contributed unique variance in single word reading and passage comprehension, but not non-word reading.

Table 2

Bivariate correlations for SES, PA, non-word reading, single word reading, and passage comprehension

\begin{tabular}{|c|c|c|c|c|c|c|}
\hline Measure & SES & $\begin{array}{l}\text { Phonological } \\
\text { awareness }\end{array}$ & $\begin{array}{l}\text { Non-word } \\
\text { reading }\end{array}$ & $\begin{array}{l}\text { Single word } \\
\text { reading }\end{array}$ & $\begin{array}{l}\text { Passage } \\
\text { comprehension }\end{array}$ & PPVT \\
\hline SES & - & 0.441 & 0.376 & 0.415 & 0.510 & 0.663 \\
\hline Phonological awareness & & - & 0.679 & 0.673 & 0.650 & 0.571 \\
\hline Non-word reading & & & - & 0.859 & 0.733 & 0.400 \\
\hline Single word reading & & & & - & 0.852 & 0.433 \\
\hline Passage comprehension & & & & & - & 0.556 \\
\hline
\end{tabular}

Note: All correlations are significant at the $p<0.0001$ level. PPVT: peabody picture vocabulary test, 3rd edition. 
Table 3

Hierarchical regressions predicting non-word reading, single word reading and passage comprehension in the whole sample

\begin{tabular}{lllllll}
\hline Task & Model & $R^{2}$-change & $B$ & S.E. $B$ & Beta & $p$ \\
\hline Non-word reading & PA & 0.493 & 4.3 & 0.36 & 0.702 & 0.0001 \\
& PA & 0.006 & 4.1 & 0.40 & 0.666 & 0.0001 \\
& SES & & 1.4 & 1.1 & 0.083 & 0.207 \\
Single word reading & PA & 0.47 & 4.4 & 0.39 & 0.686 & 0.0001 \\
& PA & 0.016 & 4.0 & 0.42 & 0.624 & 0.0001 \\
& SES & & 2.5 & 1.1 & 0.142 & 0.034 \\
Passage comprehension & PA & 0.449 & 3.1 & 0.28 & 0.670 & 0.0001 \\
& PA & 0.058 & 2.6 & 0.30 & 0.553 & 0.0001 \\
& SES & & 3.3 & 0.81 & 0.268 & 0.0001 \\
\hline
\end{tabular}

Note: PA: phonological awareness composite; SES: socioeconomic status composite.

\subsubsection{Testing for an interaction: distribution of SES across PA}

Although PA and SES could each explain variance in single word reading and comprehension, the absence of a main effect in non-word reading would be uninterpretable in the presence of an interaction (Pedhazur, 1997). However, to determine whether differences in reading achievement associated with SES were independent of or interacting with differences in PA, the range of SES in the sample must be represented across all levels of PA. In fact, this was not the case in the whole sample. For instance, no children from the highest tercile of SES scored at the lowest PA levels. This reflects the fact that SES and PA are themselves correlated (Dickinson \& Snow, 1987; Noble et al., 2005; Wallach et al., 1977; Whitehurst, 1997).

To test for an interaction it was therefore necessary to restrict the data to a subset in which children from diverse SES backgrounds scored within an overlapping range of PA. An adequately broad range of SES was represented between the scores of 7 and 13 on the PA composite, i.e. one standard deviation above and below the nationally standardized mean of 10 for the CTOPP subtests. Interaction analyses are thus limited to the subset of children who scored within the average range of PA. This subset of 116 children did not differ from the full sample in SES (mean 0.13 , S.D. $0.92, p<0.14$ ).

Using only participants scoring within the average range of PA, hierarchical regressions were conducted in which the PA composite and the SES composite were entered in the first step, followed by the SES $\times$ PA interaction. Results are presented in Table 4. Note that, although the variance explained at the first step is generally reduced due to the inclusion of fewer subjects (and resultant loss of power), a similar pattern holds. Specifically, SES accounts for unique variance in the second step for both single word reading and passage comprehension. Interestingly, however, the PA $\times$ SES interaction was significant for non-word reading. Thus, without investigating this interaction, we would have mistakenly concluded that SES does not systematically relate to nonword decoding skills.

It should be noted that reducing the data set in this manner yields a more conservative test of the interaction. If the interaction analysis is (inappropriately) run across the whole group, more variance is accounted for by the interaction term.

The relationships between SES, PA and each reading measure are represented schematically in Fig. 1, using children from the average PA range. Reading scores for all three reading tasks are plotted against PA, with the continuum of the SES composite schematically represented by 


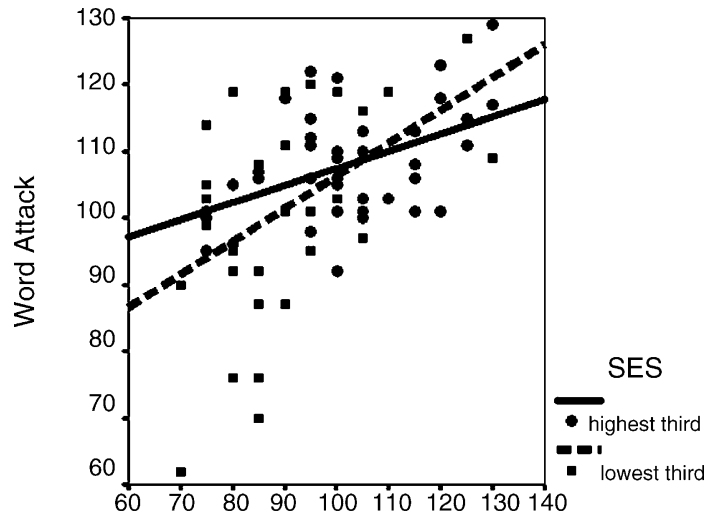

Phonological Awareness Composite

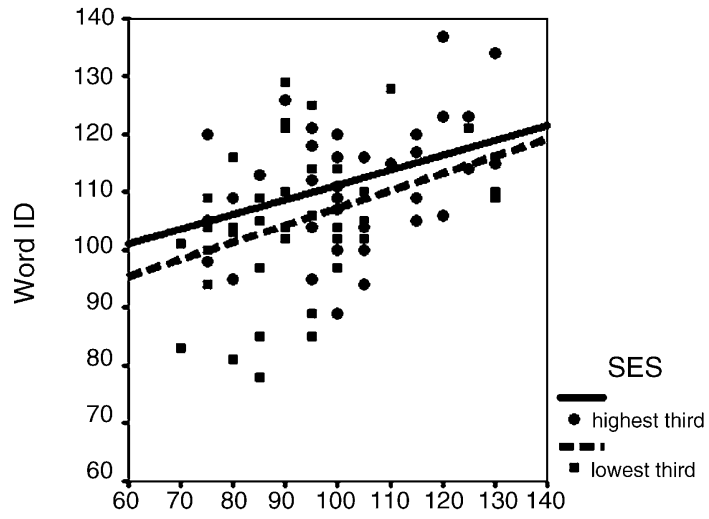

Phonological Awareness Composite

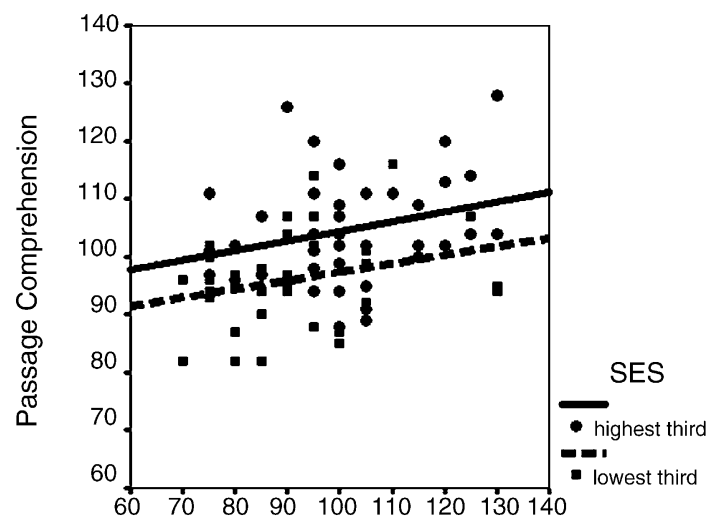

Phonological Awareness Composite

Fig. 1. Non-word reading (top), single word reading (middle), and passage comprehension (bottom) plotted against phonological awareness. Main effects and interactions with the continuous variable of SES are schematically rendered using the top and bottom terciles of SES, and plotting the regression lines (phonological awareness vs. reading measure) separately for each SES tercile, for each task. 
Table 4

Hierarchical regressions predicting non-word reading, single word reading and passage comprehension in children with average PA

\begin{tabular}{|c|c|c|c|c|c|c|}
\hline Task & Model & $R^{2}$-change & $B$ & S.E. $B$ & Beta & $p$ \\
\hline \multirow[t]{6}{*}{ Non-word reading } & PA & 0.279 & 4.0 & 0.61 & 0.528 & 0.0001 \\
\hline & PA & 0.011 & 3.8 & 0.62 & 0.500 & 0.0001 \\
\hline & SES & & 1.4 & 1.0 & 0.108 & 0.191 \\
\hline & $\mathrm{PA}$ & 0.030 & 4.0 & 0.62 & 0.521 & 0.0001 \\
\hline & SES & & 17.3 & 7.2 & 1.361 & 0.018 \\
\hline & $\mathrm{PA} \times \mathrm{SES}$ & & -1.6 & 0.74 & -1.271 & 0.028 \\
\hline \multirow[t]{6}{*}{ Single word reading } & PA & 0.161 & 3.1 & 0.65 & 0.401 & 0.0001 \\
\hline & PA & 0.033 & 2.7 & 0.67 & 0.353 & 0.0001 \\
\hline & SES & & 2.4 & 1.1 & 0.187 & 0.035 \\
\hline & PA & 0.009 & 2.8 & 0.67 & 0.364 & 0.0001 \\
\hline & SES & & 10.9 & 7.8 & 0.855 & 0.167 \\
\hline & $\mathrm{PA} \times \mathrm{SES}$ & & -.87 & 0.80 & -0.677 & 0.275 \\
\hline \multirow[t]{6}{*}{ Passage comprehension } & $\mathrm{PA}$ & 0.133 & 2.2 & 0.53 & 0.365 & 0.0001 \\
\hline & PA & 0.115 & 1.7 & 0.51 & 0.274 & 0.002 \\
\hline & SES & & 3.6 & 0.86 & 0.352 & 0.0001 \\
\hline & PA & 0.0001 & 1.7 & 0.52 & 0.276 & 0.002 \\
\hline & SES & & 5.1 & 6.1 & 0.507 & 0.399 \\
\hline & $\mathrm{PA} \times \mathrm{SES}$ & & -.16 & 0.62 & -0.157 & 0.795 \\
\hline
\end{tabular}

Note: PA: phonological awareness composite; SES: socioeconomic status composite.

the top and bottom terciles. A strong main effect of PA is evident in all three reading tasks, as performance increases across the board as PA improves. A main effect of SES is also seen, with higher SES generally associated with higher performance at a given level of PA. Finally, an $\mathrm{SES} \times \mathrm{PA}$ interaction is evident in non-word reading. Post-hoc analysis reveals that the difference in non-word reading across SES is most pronounced at lower PA levels. Specifically, SES accounts for variance in non-word reading scores of children who scored below the national average of 100 on the PA composite $\left(R^{2}=0.11 ; p<0.01\right)$, whereas SES did not account for variance in non-word reading among children who scored above the national PA average $\left(R^{2}=0.007 ; p<0.53\right)$. Thus, in the lower half of PA, there is a difference in non-word reading across SES. In the higher half of PA, there is no difference in non-word reading across SES.

\subsection{Receptive vocabulary}

SES, representing a composite of parental education, occupation, and income-to-needs, is a proxy measure for a number of conditions and experiences that may underlie cognitive development. Thus far, we have replicated the literature showing that SES is associated with reading, and have extended it by showing that SES interacts with PA to predict decoding. Given that SES disparities are associated with disparities in verbal language skills (e.g. Hoff, 2003), and that verbal abilities have been partially (Raz \& Bryant, 1990) or completely (Bowey, 1995) shown to account for SES disparities in various reading skills, we explored whether general verbal ability could account 
for the association between SES and reading. Regressions similar to those above were conducted, with the inclusion of the PPVT along with PA and SES. The inclusion of receptive vocabulary partially reduced the variance in reading ability explained by SES. In non-word reading and single word reading, PPVT did not explain any unique variance. However, whereas SES had previously explained significant variance in single word reading performance when accounting for only PA, now the variance accounted for by SES was only marginally significant when controlling for both PA and verbal skills $($ Beta $=0.148 ; p<0.065)$. In passage comprehension, PPVT did uniquely explain a portion of the variance when accounting for PA and SES (Beta $=0.196 ; p<0.022$ ). However, SES continued to account for significant unique variance (Beta $=0.179 ; p<0.021)$.

When performing the same analysis among participants who scored within the average PA range, the same pattern of results was observed, and there remained an SES $\times$ PA interaction for non-word reading. ${ }^{1,2}$ Again, had we neglected to include the interaction term, we would have mistakenly concluded that SES does not systematically relate to non-word reading performance.

\section{Discussion}

Socioeconomic background plays an important role predicting in early reading ability, even when controlling for PA skill. This underscores the fact that multiple factors play a role in the development of reading, and in predicting whether a child will be a successful reader or will instead have difficulty in acquiring this skill that is so crucial for academic and life achievement (McCardle, Scarborough, \& Catts, 2001).

When non-word reading is examined in the subset of children whose PA fell within a range constrained by data from across the SES spectrum - thus rendering the subset suitable for the exploration of interaction effects - a significant SES $\times$ PA interaction emerges. In contrast to the relationship observed in non-word reading, SES explains a small amount of unique additive variance in real word reading when controlling for PA. Controlling for vocabulary eliminates this effect, suggesting that vocabulary differences may mediate the SES association with single word reading. In passage comprehension, SES also explains some unique variance after controlling for PA. However, in this case, covarying vocabulary reduces, but does not eliminate, the SES association with this skill. No SES $\times$ PA interaction was observed for either real word reading or passage comprehension, and in both cases the standardized regression coefficients reveal that both SES and PA play a large role.

\footnotetext{
${ }^{1}$ We also re-ran these regressions, excluding the 32 children who had a significant medical or psychiatric history. Results were remarkably similar: in both non-word reading and single word reading, neither the PPVT nor SES explained significant unique variance when also accounting for PA, as in the full sample. In passage comprehension, significant variance was again explained by the PPVT when accounting for PA and SES. However, unlike in the full sample, SES was only marginally significant $(p<0.10)$, though this may be due to reduced power due to eliminating participants. The same pattern of results was observed for the analysis of participants within the average range of PA; as before, the SES $\mathrm{x}$ PA interaction remained significant for non-word reading.

2 Although PA did not differ on average as a function of whether a child was strictly monolingual or had some second language exposure $(t(148)=1.07 ; p<0.29)$, we nonetheless examined what effect, if any, second language exposure may have on reading abilities even after accounting for PA and general verbal ability. Second language exposure did not explain unique variance in any of the three reading measures, when accounting for PA and PPVT. In single word reading, SES accounted for a slight amount of variance when covarying PA, PPVT and second language exposure. It is thus unlikely that second language exposure is associated with the unique variance in single word reading explained by SES, as SES was previously only marginally significant. If anything, language exposure appears to have been slightly masking an SES effect in single word reading. The same analyses among children in the average range of PA yielded similar results, and again the SES $\times$ PA interaction accounted for additional variance in non-word reading.
} 


\subsection{Models of influence}

Beyond the well-established link in the cognitive literature between early PA and later reading ability (e.g. Adams, 1990; L. Bradley \& Bryant, 1983; Wagner \& Torgesen, 1987), there are additional factors - for which SES stands as a proxy variable - that help to determine whether a child will successfully learn to read.

To explain the complex relationships between SES, PA, and reading outcome, we posited a "Multiplicative Factors Hypothesis," which predicted that SES would modulate the association between PA and reading skill. This model captured an important aspect of the data presented for non-word reading. The top panel of Fig. 1 reveals that, among children whose PA is within a standard deviation above or below the national average, SES systematically influences the relationship between PA and decoding ability. Post-hoc testing confirmed that the disparity in decoding ability across SES is pronounced among children at the lower end of the PA spectrum. At higher phonological skill levels, however, the differences in decoding across SES diminish to non-significant levels, such that SES no longer accounts for variance in non-word reading skill among children who have above average PA. That is, regardless of background, children with higher PA abilities are decoding successfully. It is only among children with relatively lower PA levels that we see a strong disparity in decoding ability across SES. The influences of PA and SES on decoding are thus multiplicative, amplifying one another when predicting low decoding achievement. Importantly, without investigating the presence of such an interaction, we would have incorrectly rejected the conclusion that SES has a systematic relationship with non-word reading. In light of this, any study that fails to find unique variance accounted for by SES without exploring this interaction is in effect rendered uninterpretable, in the sense that it cannot distinguish between a case in which no SES contribution exists versus a case in which a different SES contribution exists for different levels of PA.

Two alternate models were also considered. The "Mediating Factors Model" states that PA directly influences reading ability, but that SES affects reading only indirectly through its impact on this cognitive precursor to reading. Some evidence was found for this model. PA and SES were themselves highly correlated, and there was a large degree of shared variance between PA and SES in predicting all reading measures. This is evidenced by the fact that, although the bivariate correlations between SES and each measure of reading achievement were quite high, there was a comparatively small increase in variance accounted for by SES when controlling for PA. In other words, much of the association between SES and reading can be statistically accounted for by differences in PA skill. Importantly, in a cross-sectional design, only statistical mediation may be examined - that is, it is impossible to know whether, mechanistically, PA mediates the effect of SES on reading outcome, or conversely, whether SES differences mediate the effect of PA on reading.

Finally, the "Independent Factors Model" states that SES and PA each exert independent effects on reading. Supporting this, SES did account for unique, additive variance in both single word reading and passage comprehension after statistically controlling for PA. That is, across a range of PA levels, SES has a consistent association with both single word reading and reading comprehension, such that children from higher SES backgrounds are consistently at an advantage, as illustrated by the relatively parallel lines in the second and third panels of Fig. 1.

Throughout our analyses, we observed no SES $\times$ PA interaction for either real word reading or passage comprehension. Although future research is necessary to investigate why this is the case, it stands to reason that reading skills that rely heavily on PA would most powerfully demonstrate an interaction, whereas reading skills that rely on other factors would not, given the same sample 
size. That is, reading skills in which PA plays a progressively smaller proportionate role - but in which other factors such as vocabulary or print exposure play a larger role - would have less variance accounted for by PA, and would therefore have less variance available to demonstrate the interaction. Non-word reading has traditionally been regarded as the most basic measure of the child's ability to apply PA, relative to other types of reading skills (Share, 1995).

Alternatively, the application of phonological awareness to grapheme-phonemecorrespondence - as is necessary for non-word decoding - is enhanced by formal teaching, whereas other aspects of reading development may be less so. As schooling is an excellent predictor of early elementary reading skill (Morrison, Griffith, \& Alberts, 1997), the specificity of the interaction could potentially be explained if children from different SES levels react to formal teaching differently (e.g. Morrison \& Connor, 2002), or perhaps higher quality formal teaching is simply more likely among higher SES children (e.g. Ramey \& Ramey, 1998). Other reading skills may rely less on formal teaching, and more on print exposure or vocabulary development, which may show more uniform differences across SES (Raz \& Bryant, 1990).

\subsection{Implications for developmental research}

Understanding the precursors of early reading is an important goal for developmental psychology. The two best established predictors - phonological awareness and socioeconomic status have been studied extensively, but almost always in isolation from one another. As developmental scientists, we must recognize that the cognitive and experiential factors driving development interact in potentially more complex ways than have been acknowledged, at least in studies of reading development. SES is not a simple construct that necessarily combines additively with other influences on cognitive development. Rather, we have shown that it can buffer against or synergize with other influences in a multiplicative fashion, varying its effects as a function of other child characteristics. These statements have potentially far-reaching implications, both within and beyond the field of reading research. Too often, SES is neglected as a factor in investigating cognitive development. Although studying a socioeconomically uniform population may eliminate confounds, it also eliminates the possibility of understanding how a cognitive process generalizes across the population. In the case of non-word decoding, an investigator cannot assess the effects of PA without regard to SES. Given the extensive literature linking numerous aspects of cognitive development to SES, this point is likely to extend beyond the study of reading.

The assumption of independent, additive influences on development makes the analysis of developmental mechanisms relatively simple. However, the causes of, and limitations on, achievement are apparently interactive and therefore more complex. If we are to understand the development of reading, and potentially other abilities, we must acknowledge the multiplicity of interacting factors, including socioeconomic status, and begin to unpack this complex construct.

The complex social and economic correlates of SES - including home environment (BrooksGunn et al., 1996; Jackson et al., 2000; Korenman et al., 1995), and early education factors (Barnett, 1998; Ramey \& Ramey, 1998) - likely directly influence cognitive skills important in the development of reading. By incorporating relatively objective measures that involve direct home and school observation (R.H. Bradley, Corwyn, Pipes McAdoo et al., 2001; Morrison et al., 1997), future research may shed further light on the means by which SES differences modulate the relationship between PA and reading achievement.

Our study cannot differentiate between genetic and environmental factors for which SES may stand as a proxy. Interestingly, however, several studies have shown that genetic factors are more strongly related to language development or IQ among children from higher SES families, 
whereas environmental factors are more strongly predictive of these cognitive abilities among children from lower SES backgrounds (Rowe, Jacobson, \& van den Oord, 1999; Turkheimer, Haley, Waldron, D'Onofio, \& Gottesman, 2003). This has been interpreted as suggesting that the genetic potential for learning is expressed more fully when children have plentiful resources (Rowe et al., 1999). Our findings of a narrower range of non-word reading abilities observed among higher SES children would fit with this notion.

Finally, striking differences in SES-PA-reading relationships have been observed across development (Hecht et al., 2000; Raz \& Bryant, 1990). Although this sample represented a crosssectional study of a single age group, future studies should examine the SES $\times$ PA interaction across development, with particular attention to changes that occur from kindergarten through the second or third grade, a period of robust reading development. This would allow for a better assessment of preliterate skills, as well as a more reliable measure of reading outcome and disability. In addition, the inclusion of bilingual children would potentially increase the generalizability of the findings.

\subsection{Implications for education}

The relationship between PA and reading ability has had a large impact on education policy (Report of the National Reading Panel, 2000). The present results underscore this relationship, as PA is a robust predictor of all reading measures investigated. However, it is imperative that the effects of such cognitive variables be examined in the context of a child's socioeconomic background, if we are to formulate well-grounded education policies that are generalizable to many different populations.

SES modulates the relationship between PA and decoding skill, and this interaction has several important implications for education. First, at higher skill levels, the association between SES and decoding diminishes. That is, regardless of background, a child with relatively high PA abilities will likely learn to decode successfully. Secondly, the association between low PA and low decoding skill is amplified in a lower SES environment. It is therefore critical that cognitive risk factors be appropriately addressed in such children. In fact, a child with lower PA who comes from the lower end of the socioeconomic spectrum may actually have multiple interventional options. If the present findings are representative of a causal relationship between PA and reading, then training in PA and closely related constructs such as letter-sound mapping may actually prove to be more effective for children struggling with decoding who come from lower SES environments. In particular, we would predict greater increases in reading standard scores per unit growth in PA, as suggested by the steeper slopes for the lowest SES tercile in the first panel of Fig. 1. In addition, interventions that target the large disparities in experiences associated with SES - experiences such as health care, early education, and home environment that likely play at least a partially etiological role in the achievement gap across SES (Bornstein \& Bradley, 2003) - may serve to reduce this experience differential, and thus reduce the ensuing gap in achievement. That is, Fig. 1 suggests the possibility that children from lower SES environments who are given opportunities more in line with those received by their higher SES peers may actually be "shifted" to a less steep curve such that, for a given level of low PA, the associated decoding ability might be higher. It should be noted that, strictly speaking, the results presented here cannot distinguish between a situation in which higher SES "buffers" lower phonological skill, or one in which lower SES children with higher PA are performing unexpectedly well. In support of the first possibility, however, Telzrow (1987) found that higher socioeconomic background may attenuate the longterm academic difficulties of children with learning disabilities, with or without intervention. In 
addition, changing a child's circumstances may result in improvement across diverse domains, ranging from cognitive performance to mental health (Costello, Compton, Keeler, \& Angold, 2003; Leventhal \& Brooks-Gunn, 2003a).

Of course, although basic decoding skills are certainly necessary for the development of fluent word reading and comprehension abilities, it is these more complex skills that are ultimately necessary for the successful acquisition of literacy. In our sample, these skills had similar associations with SES across the PA spectrum, such that children from lower socioeconomic backgrounds were consistently at a disadvantage in terms of reading outcome, regardless of basic phonological skill. It must therefore be ensured that all children have the necessary opportunities to succeed. Among children struggling with reading, the most effective programs will likely combine the training of PA and related constructs with environmental interventions designed to target the factors that lead to disparities in experience across SES. The challenge, of course, is to determine which of these disparities may be most effectively targeted, and how such targeting may best serve the millions of children for whom socioeconomic disadvantage places them at risk for low achievement-a challenge that has been repeatedly confronted by scores of sociological, educational, medical and developmental psychological researchers (see Shonkoff \& Meisels, 2000).

\section{Conclusions}

SES has a systematic effect on reading skills, after controlling for PA. However, the nature of this effect changes, depending on both the reading measure and the PA range examined. SES disparities in decoding are greatest at lower PA levels, whereas SES disparities in single word reading and passage comprehension are consistent across all PA levels. Interventions for poor readers must therefore target both the development of phonological skill and the elimination of key discrepancies in environmental advantage. Attempts to identify the cognitive and experiential factors driving development must likewise acknowledge the potentially complex, synergizing relations between these factors.

\section{Acknowledgements}

Support for this work was provided by NIH grants R01-HD043078, R21-DA015856, R01DA20011, R01-DA014129, P50-HD25802-13, T32-MH17168, NSF grant REC-0337715, and the John Merck Scholars Program in the Biology of Developmental Disabilities in Children. We gratefully acknowledge the helpful suggestions of Sharon Thompson-Schill, Frank Furstenberg and Andy Leon. This work was conducted in partial fulfillment of the requirements for a doctoral dissertation in the Neuroscience graduate program at the University of Pennsylvania.

\section{References}

Adams, M. J. (1990). Learning to read: Thinking and learning about print. Cambridge, MA: MIT press.

Barnett, W. S. (1998). Long-term cognitive and academic effects of early childhood education on children in poverty. Preventive Medicine, 27, 204-207.

Baydar, N., Brooks-Gunn, J., \& Furstenberg, F. (1993). Early warning signs of functional illiteracy: Predictors in childhood and adolescence. Child Development, 64, 815-829.

Bornstein, M. H., \& Bradley, R. H. (2003). Socioeconomic status, parenting, and child development. Mahwah, NJ: Lawrence Erlbaum Associates.

Bowey, J. A. (1995). Socioeconomic status differences in preschool phonological sensitivity and first-grade reading achievement. Journal of Educational Psychology, 87(3), 476-487. 
Bradley, L., \& Bryant, P. E. (1983). Categorizing sounds and learning to read-A causal connection. Nature, 301, 419421.

Bradley, R. H., Corwyn, R. F., Burchinal, M., Pipes McAdoo, H., \& Garcia Coll, C. (2001). The home environments of children in the United States. Part II. Relations with behavioral development through age thirteen. Child Development, 72(6), 1868-1886.

Bradley, R. H., Corwyn, R. F., Pipes McAdoo, H., \& Garcia Coll, C. (2001). The home environments of children in the United States. Part I. Variations by age, ethnicity and poverty status. Child Development, 72(6), 1844-1867.

Brooks-Gunn, J., Guo, G., \& Furstenberg, F. (1993). Who drops out of and who continues beyond high school? Journal of Research on Adolescence, 3, 271-294.

Brooks-Gunn, J., Klebanov, P. K., \& Duncan, G. J. (1996). Ethnic differences in children's intelligence test scores: role of economic deprivation, home environment, and maternal characteristics. Child Development, 67, 396-408.

Byrne, B., Fielding-Barnesley, R., \& Ashley, L. (2000). Effects of preschool phoneme identity training after six years: Outcome level distinguished from rate of response. Journal of Educational Psychology, 92(4), 659-667.

Cornwall, A. (1992). The relationship of phonological awareness, rapid naming, and verbal memory to severe reading and spelling disability. Journal of Learning Disabilities, 25(8), 532-538.

Costello, E. J., Compton, S. N., Keeler, G., \& Angold, A. (2003). Relationships between poverty and psychopathology: A natural experiment. Journal of the American Medical Association, 290, 2023-2029.

Dickinson, D. K., \& Snow, C. E. (1987). Interrelationships among prereading and oral language skills in kindergartners from two social classes. Early Childhood Research Quarterly, 2., 1-25.

Ensminger, M. E., \& Fothergill, K. E. (2003). A decade of measuring SES: What it tells us and where to go from here. In M. H. Bornstein \& R. H. Bradley (Eds.), Socioeconomic status, parenting and child development (pp. 13-27). Mahwah, New Jersey: Lawrence Erlbuaum Associates.

Fisher, S. E., \& DeFries, J. C. (2002). Developmental dyslexia: Genetic dissection of a complex cognitive trait. Nature Reviews Neuroscience, 3, 767-780.

Foorman, B. R., Francis, D. J., Novy, D. M., \& Liberman, D. (1991). How letter-sound instruction mediates progress in first-grade reading and spelling. Journal of Educational Psychology, 83(4), 456-469.

Gottfried, A. W., Gottfried, A. E., Bathurst, K., Guerin, D. W., \& Parramore, M. M. (2003). Socioeconomic status in children's development and family environment: infancy through adolescence. In M. H. Bornstein \& R. H. Bradley (Eds.), Socioeconomic status, parenting and child development (pp. 189-207). Mahwah, New Jersey: Lawrence Erlbaum Associates.

Hawley, T., \& Disney, E. (1992). Crack's children: The consequences of maternal cocaine abuse. Social Policy Report, 6 , $1-22$.

Hecht, S. A., Burgess, S. R., Torgesen, J. K., Wagner, R. K., \& Rashotte, C. A. (2000). Explaining social class differences in growth of reading skills from beginning kindergarten through fourth grade: The role of phonological awareness, rate of access, and print knowledge. Reading and Writing, 12(1-2), 99-127.

Hoff, E. (2003). Causes and consequences of SES-related differences in parent-to-child speech. In M. H. Bornstein \& R. H. Bradley (Eds.), Socioeconomic status, parenting and child development (pp. 145-160). Mahwah, NJ: Lawrence Erlbaum Associates.

Hollingshead, A. B. (1975). Four factor index of social status. New Haven: Yale University Department of Sociology.

Jackson, A. P., Brooks-Gunn, J., Huang, C., \& Glassman, M. (2000). Single mothers in low-wage jobs: Financial strain, parenting and preschoolers' outcomes. Child Development, 71, 1409-1423.

Klein, N., Hack, M., \& Breslau, N. (1989). Children who were very low birthweight: Development and academic achievement at nine years of age. Journal of Developmental and Behavioral Pediatrics, 10, 32-37.

Knopnik, V. S., Smith, S. D., Cardon, L., Pennington, B., Gayan, J., Olson, R. K., et al. (2002). Differential genetic etiology of reading component processes as a function of IQ. Behavior Genetics, 32(3), 181-198.

Korenman, S., Miller, J., \& Sjaastad, J. (1995). Long-term poverty and child development in the United States: Results from the NLSY. Children and Youth Services Review, 17, 127-155.

Leventhal, T., \& Brooks-Gunn, J. (2003a). Children and youth in neighborhood contexts. Current Directions in Psychological Science, 12(1), 27-31.

Leventhal, T., \& Brooks-Gunn, J. (2003b). Moving on up: Neighborhood effects on children and families. In M. H. Bornstein \& R. H. Bradley (Eds.), Socioeconomic status, parenting and child development (pp. 209-230). Mahwah, New Jersey: Lawrence Erlbaum Associates.

Liaw, F.-R., \& Brooks-Gunn, J. (1994). Cumulative familial risks and low-birthweight children's cognitive and behavioral development. Journal of Clinical Child Psychology, 23(4), 360-372.

Lonigan, C., Burgess, S., Anthony, J., \& Barker, T. (1998). Development of phonological sensitivity in 2- to 5-year-old children. Journal of Educational Psychology, 90, 294-311. 
MacDonald, G. W., \& Cornwall, A. (1995). The relationship between phonological awareness and reading and spelling achievement eleven years later. Journal of Learning Disabilities, 28(8), 523-527.

McCandliss, B. D., \& Noble, K. G. (2003). The development of reading impairment: A cognitive neuroscience model. Mental Retardation and Developmental Disabilities Research Reviews, 9, 196-204.

McCardle, P., Scarborough, H. S., \& Catts, H. W. (2001). Predicting, explaining, and preventing children's reading difficulties. Learning Disabilities Research and Practice. Special Issue: Emergent and early literacy: Current Status and Research Directions, 16(4), 230-239.

McCormick, M. (1989). Long-term follow-up of infants discharged from the neonatal intensive care unit. Journal of the American Medical Association, 261, 1767-1772.

McLoyd, V. C. (1998). Socioeconomic disadvantage and child development. American Psychologist, 53(2), 185204.

Morrison, F. J., \& Connor, C. M. (2002). Understanding schooling effects on early literacy: A working research strategy. Journal of School Psychology, 40(6), 493-500.

Morrison, F. J., Griffith, E. M., \& Alberts, D. M. (1997). Nature-nurture in the classroom: entrance age school readiness, and learning in children. Developmental Psychology, 33(2), 254-262.

Needleman, H. L., Schell, A., Bellinger, D., Leviton, A., \& Allred, E. (1990). The long-term effects of low doses of lead in childhood: An eleven-year followup report. New England Journal of Medicine, 322, 83-88.

Noble, K. G., Norman, M. F., \& Farah, M. J. (2005). Neurocognitive correlates of socioeconomic status in kindergarten children. Developmental Science, 8(1), 74-87.

Pedhazur, E. J. (1997). Multiple categorical independent variables and factorial designs. In Multiple regression in behavioral research: Explanation and prediction. South Melbourne, Australia: Wadsworth Thomson Learning.

Ramey, C., \& Ramey, S. (1998). Prevention of intellectual disabilities: Early interventions to improve cognitive development. Preventive Medicine, 27, 224-232.

Raz, I. S., \& Bryant, P. (1990). Social background, phonological awareness and children's reading. British Journal of Developmental Psychology, 8(3), 209-225.

Report of the National Reading Panel. (2000). Washington, DC: National Institute of Child Health and Human Development.

Rowe, D. C., Jacobson, K. C., \& van den Oord, E. J. C. G. (1999). Genetic and environmental influences on vocabulary IQ: Parental education level as moderator. Child Development, 70(5), 1151-1162.

Share, D. L. (1995). Phonological recoding and self-teaching: Sine que non of reading acquisition. Cognition, 55(2), 151-218.

Share, D. L., Jorm, A. F., MacLean, R., \& Mathews, R. (1984). Sources of individual differences in reading acquisition. Journal of Educational Psychology, 76, 1309-1324.

Shonkoff, J. P., \& Meisels, S. L. (Eds.). (2000). Handbook of early childhood intervention. Cambridge, UK: Cambridge University Press.

Smith, J., Brooks-Gunn, J., \& Klebanov, P. (1997). Consequences of living in poverty for young children's cognitive and verbal ability and early school achievement. In G. Duncan \& J. Brooks-Gunn (Eds.), Consequences of growing up poor. New York: Russell Sage.

Telzrow, C. F. (1987). The So what? question: Intervention with learning disabled children. In J. M. Williams \& C. J. Long (Eds.), The rehabilitation of cognitive disabilities (pp. 191-205). New York: Plenum Press.

Torgesen, J. K., Alexander, A. W., Wagner, R. K., Rashotte, C. A., Voeller, K. K., Conway, T., et al. (2001). Intensive remedial instruction for children with severe reading disabilities: Immediate and long-term outcomes from two instructional approaches. Journal of Learning Disabilities, 34.

Turkheimer, E., Haley, A., Waldron, M., D’Onofio, B., \& Gottesman, I. I. (2003). Socioeconomic status modifies heritability of IQ in young children. Psychological Science, 14(6), 623-628.

Vellutino, F. R., Scanlon, D., Sipay, E. R., Pratt, A., Chen, R., \& Denckla, M. B. (1996). Cognitive profiles of difficult-toremediate and readily remediated poor readers: Early intervention as a vehicle for distinguishing between cognitive and experiential deficits as basic causes of a specific reading disability. Journal of Educational Psychology, 88, 601-638.

Wagner, R. K., \& Torgesen, J. K. (1987). The nature of phonological processing and its causal role in the acquisition of reading skills. Psychological Bulletin, 101, 192-212.

Wagner, R. K., Torgesen, J. K., \& Rashotte, C. A. (1999). Comprehensive test of phonological processing. Austin, TX: Pro-Ed.

Wagner, R. K., Torgesen, J. K., Rashotte, C. A., Hecht, S. A., Barker, T. A., Burgess, S. R., et al. (1997). Changing relations between phonological processing abilities and word-level reading as children develop from beginning to skilled readers: A 5-year longitudinal study. Developmental Psychology, 33(3), 468-479. 
Wallach, L., Wallach, M. A., Dozier, M. G., \& Kaplan, N. E. (1977). Poor children learning to read do not have trouble with auditory discrimination but do have trouble with phoneme recognition. Journal of Educational Psychology, 69, 36-39.

White, K. R. (1982). The relation between socio-economic status and academic achievement. Psychological Bulletin, 91 , $461-481$.

Whitehurst, G. J. (1997). Language processes in context: Language learning in children reared in poverty. In L. B. Adamson \& M. A. Romski (Eds.), Research on communication and language disorders: contribution to theories of language development (pp. 233-266). Baltimore: Brookes.

Woodcock, R. W., McGrew, K. S., \& Mather, N. (2001). WJ III tests of cognitive abilities. Itasca, IL: Riverside Publishing. 